\title{
GLOBAL ANALYSIS OF A COCIRCULATING TARGET CELLS HIV MODEL WITH DIFFERENTIAL DRUG EFFICACY AND NONLINEAR INCIDENCE RATE
}

\author{
A. M. ELAIW AND N. A. ALMUALLEM
}

This paper is dedicated to the memory of Professor Miklós Farkas.

\author{
Received 15 June, 2014
}

\begin{abstract}
The main purpose of this work is to investigate the qualitative behavior of an HIV dynamics model with two types of cocirculating target cells. The model takes into account both short-lived and long lived chronically infected cells. In the two types of target cells, the drug efficacy is assumed to be different. The incidence rate is represented by Crowley-Martin functional response. First we have derived the basic reproduction number $R_{0}$, then constructed Lyapunov functions to establish the global asymptotic stability of the disease-free and endemic equilibria of the model. We have been proven that, the disease-free equilibrium is globally asymptotically stable (GAS) when $R_{0} \leq 1$, and the endemic equilibrium is GAS when $R_{0}>1$. Numerical simulations have been carried out to support our theoretical results.
\end{abstract}

2010 Mathematics Subject Classification: 34D20; 34D23; 37N25; 92D30

Keywords: HIV infection, global dynamics, chronically infected cells, Lyapunov method

\section{INTRODUCTION}

Although the mathematical modeling of human immunodeficiency virus (HIV) dynamics alone cannot deals with issues associated with HIV infection, it can be a helpful tool for a good understanding the viral dynamics in vivo. Mathematical models can improved strategies of diagnosis and treatment. Today, many anti-HIV drugs are available for patients with HIV, which led to a rapid decrease in viruses and an increase in the major target cells of the virus, $\mathrm{CD}^{+} \mathrm{T}$ cells. There are two main categories of anti-HIV, the reverse-transcriptase inhibitors RTIs drugs which prevent HIV from infecting the target cells, and the protease inhibitors PIs drugs which prevent the infected cells from producing new infectious viruses [1]. In the literature, several mathematical models describing the HIV dynamics in vivo have been proposed (see, e.g. [3], [6-10], [12,13]). However, these models did not take into account the difference between short-lived infected cells and long lived chronically infected cells. In HIV infection, the short-lived infected cells produce much larger 
amounts of viruses than long lived chronically infected cells and die at a much faster rate [2]. The basic HIV infection model which takes into account long lived chronically infected cells under the effect of antiviral drug therapy has been proposed in [2] as:

$$
\begin{aligned}
& \dot{x}=\lambda-d x-(1-\varepsilon) \bar{\beta} x v, \\
& \dot{y}=(1-q)(1-\varepsilon) \bar{\beta} x v-\delta y, \\
& \dot{z}=q(1-\varepsilon) \bar{\beta} x v-a z, \\
& \dot{v}=N \delta y+M a z-u v,
\end{aligned}
$$

where $x, y, z$ and $v$ represent, respectively, the concentrations of the uninfected CD4 ${ }^{+}$ $\mathrm{T}$ cells, short-lived infected cells, long lived chronically infected cells, and free viruses. Parameters $\lambda$ and $d$ are the birth rate and death rate constants of the uninfected $\mathrm{CD} 4^{+} \mathrm{T}$ cells. Parameter $\bar{\beta}$ is the infection rate constant. The short-lived infected $\mathrm{CD} 4{ }^{+} \mathrm{T}$ cells, long lived chronically infected $\mathrm{CD} 4{ }^{+} \mathrm{T}$ cells and free viruses are die with rate constants $\delta, a$ and $u$, respectively. The fractions $(1-q)$ and $q$ with $0<q<1$ are the probabilities during the infection on which an uninfected cell will become either short-lived infected or long lived chronically infected. Parameters $N$ and $M$ are the average number of virus particles generated in the lifetime of the short-lived infected and long lived chronically infected cells, respectively. The model incorporates RTI drug therapy with drug efficacy denoted by $\varepsilon$ and $0 \leq \varepsilon \leq 1$. All the parameters mentioned in the model are positive.

In a very recent work [8], model (1.1)-(1.4) has been modified to consider the antibody immune response. In model (1.1)-(1.4), it was assumed that, the HIV has one class of target cells, CD4 ${ }^{+} \mathrm{T}$ cells. Based on the observation of Perelson et al. [9] that, the HIV attack two types of cells $\mathrm{CD}^{+}{ }^{+} \mathrm{T}$ cells and macrophages. Recently, many efforts have been devoted to propose and analyze various mathematical models of HIV dynamics with two classes of target cells [2, 5, 6, 8, 10] In [4], the virus dynamics models have been studied by assuming that the virus has multiple classes of uninfected target cells. However, in [5, 6, 8, 10] and [4], only short-lived infected cells has been considered. In [2], the model (1.1)-(1.4) was extended to take into consideration two cocirculating target cells, $\mathrm{CD} 4^{+} \mathrm{T}$ cells and macrophages as:

$$
\begin{aligned}
& \dot{x}_{1}=\lambda_{1}-d_{1} x_{1}-(1-\varepsilon) \bar{\beta}_{1} x_{1} v, \\
& \dot{x}_{2}=\lambda_{2}-d_{2} x_{2}-(1-f \varepsilon) \bar{\beta}_{2} x_{2} v, \\
& \dot{y}_{1}=(1-q)(1-\varepsilon) \bar{\beta}_{1} x_{1} v-\delta y_{1}, \\
& \dot{y}_{2}=(1-q)(1-f \varepsilon) \bar{\beta}_{2} x_{2} v-\delta y_{2}, \\
& \dot{z}_{1}=q(1-\varepsilon) \bar{\beta}_{1} x_{1} v-a z_{1}, \\
& \dot{z}_{2}=q(1-f \varepsilon) \bar{\beta}_{2} x_{2} v-a z_{2},
\end{aligned}
$$




$$
\dot{v}=N \delta\left(y_{1}+y_{2}\right)+M a\left(z_{1}+z_{2}\right)-u v,
$$

where $x_{i}, y_{i}, z_{i}$, and $v$ denote the concentrations of uninfected target cells, short-lived infected cells, long lived chronically infected cells, and free viruses, respectively, and $i=1,2$ correspond to the $\mathrm{CD} 4^{+} \mathrm{T}$ cells and macrophages, respectively. In the $\mathrm{CD} 4^{+}$ T cells, the drug efficacy is $\varepsilon$ while in the macrophages the drug efficacy is $\varepsilon f$ reduced by a factor $f$ where $0<f<1$. The definitions of all parameters and variables are identical to those given in Eqs. (1.1)-(1.4).

We have observed that, the qualitative behavior of model (1.5)-(1.11) are not studied in the literature. Moreover, in model (1.5)-(1.11), the incidence rate between the uninfected target cells and viruses is given by bilinear, i.e., the infection rate per virus and per uninfected cell is constant. However, this bilinear incidence rate may not completely describe the infection process. Therefore, several forms of the incidence rate have been proposed, such as saturated incidence rate, $\frac{\beta x v}{1+\alpha v}$ where $\alpha>0$ [5], Beddington-DeAngelis functional response, $\frac{\beta x v}{1+\mu x+\alpha v}, \alpha, \mu>0$ [5], [7], [11], Crowley-Martin functional response, $\frac{\beta x v}{(1+\mu x)(1+\alpha v)}, \alpha, \mu>0$ [12], [13].

Our primary goal of the present paper is to propose an HIV dynamics model with two types of cocirculating target cells, $\mathrm{CD} 4{ }^{+} \mathrm{T}$ cells and macrophages and investigate its qualitative behavior. In the two types of target cells, the drug efficacy is assumed to be different. Both short-lived and long lived chronically infected cells are considered in the mathematical model. The incidence rate is represented by by Crowley-Martin functional response. The global stability of all equilibria of the model is established using direct Lyapunov method. The basic reproduction number $R_{0}$ of the model is derived. We have established that, the disease-free equilibrium is globally asymptotically stable (GAS) when $R_{0} \leq 1$, and the endemic equilibrium is GAS when $R_{0}>1$.

\section{THE MODEL}

In this section, we introduce a mathematical model of HIV dynamics which describes two cocirculation populations of target cells, $\mathrm{CD} 4^{+} \mathrm{T}$ cells and macrophages taking into account both of short-lived and long lived chronically infected cells. The model is more general than model (1.5)-(1.11) by assuming that the incidence rate of infection is given by Crowley-Martin functional response and the parameter $\delta, a, q, N$ and $M$ for the $\mathrm{CD}^{+}{ }^{+} \mathrm{T}$ cells and macrophages are not identically.

$$
\begin{aligned}
& \dot{x}_{i}=\lambda_{i}-d_{i} x_{i}-\frac{\beta_{i} x_{i} v}{\left(1+\mu_{i} x_{i}\right)\left(1+\alpha_{i} v\right)}, \quad i=1,2, \\
& \dot{y}_{i}=\left(1-q_{i}\right) \frac{\beta_{i} x_{i} v}{\left(1+\mu_{i} x_{i}\right)\left(1+\alpha_{i} v\right)}-\delta_{i} y_{i}, \quad i=1,2 \text {, } \\
& \dot{z}_{i}=q_{i} \frac{\beta_{i} x_{i} v}{\left(1+\mu_{i} x_{i}\right)\left(1+\alpha_{i} v\right)}-a_{i} z_{i}, \quad i=1,2,
\end{aligned}
$$




$$
\dot{v}=\sum_{i=1}^{2}\left(N_{i} \delta_{i} y_{i}+M_{i} a_{i} z_{i}\right)-u v,
$$

where $\mu_{i}, \alpha_{i}, i=1,2$ are positive constants and all the parameters and variables of the model have the same meanings as given in (1.5)-(1.11) and $\beta_{1}=(1-\varepsilon) \bar{\beta}_{1}$, $\beta_{2}=(1-f \varepsilon) \bar{\beta}_{2}$.

\section{THE ANALYSis OF THE MOdel}

One can easily show the positive invariance of the non-negative orthant $\mathbb{R}_{\geq 0}^{7}$ for model (2.1)-(2.4) (see e.g. [6] and [10]).

Proposition 1. There exist positive numbers $L_{i}, i=1,2,3$ such that the compact set $\Omega=\left\{\left(x_{1}, y_{1}, z_{1}, x_{2}, y_{2},, z_{2}, v\right) \in \mathbb{R}_{\geq 0}^{7}: 0 \leq x_{1}, y_{1}, z_{1} \leq L_{1}\right.$,

$\left.0 \leq x_{2}, y_{2}, z_{2} \leq L_{2}, 0 \leq v \leq L_{3}\right\}$ is positively invariant.

Proof. To show the boundedness of the solutions of system (2.1)-(2.4), let $G_{i}(t)=$ $x_{i}(t)+y_{i}(t)+z_{i}(t), i=1,2$ then

$$
\begin{aligned}
\dot{G}_{i}(t) & =\lambda_{i}-d_{i} x_{i}(t)-\delta_{i} y_{i}(t)-a_{i} z_{i}(t) \\
& \leq \lambda_{i}-\sigma_{i}\left(x_{i}(t)+y_{i}(t)+z_{i}(t)\right)=\lambda_{i}-\sigma_{i} G_{i}(t),
\end{aligned}
$$

where $\sigma_{i}=\min \left\{d_{i}, \delta_{i}, a_{i}\right\}$. Hence, $G_{i}(t) \leq L_{i}$, if $G_{i}(0) \leq L_{i}$ where $L_{i}=\frac{\lambda_{i}}{\sigma_{i}}$. Since $x_{i}(t), y_{i}(t)$ and $z_{i}(t)$ are all non-negative, then $0 \leq x_{i}(t), y_{i}(t), z_{i}(t) \leq L_{i}$, for all $t \geq 0$, if $0 \leq x_{i}(0)+y_{i}(0)+z_{i}(0) \leq L_{i}$. Moreover,

$$
\dot{v}=\sum_{i=1}^{2}\left(N_{i} \delta_{i} y_{i}+M_{i} a_{i} z_{i}\right)-u v \leq \sum_{i=1}^{2}\left(N_{i} \delta_{i}+M_{i} a_{i}\right) L_{i}-u v .
$$

Then $v(t) \leq L_{3}$, if $v(0) \leq L_{3}$, for all $t \geq 0$, where $L_{3}=\sum_{i=1}^{2} \frac{\left(N_{i} \delta_{i}+M_{i} a_{i}\right) L_{i}}{u}$.

Lemma 1. For system (2.1)-(2.4), there exist a threshold parameter $R_{0}>0$ such that

(i) when $R_{0} \leq 1$, there exists only one disease-free equilibrium

$$
E_{0}=\left(x_{1}^{0}, 0,0, x_{2}^{0}, 0,0,0\right) \text {, }
$$

(ii) when $R_{0}>1$, there exist $E_{0}$ and an endemic equilibrium

$E_{1}=\left(\tilde{x}_{1}, \tilde{y}_{1}, \tilde{z}_{1}, \tilde{x}_{2}, \tilde{y}_{2}, \tilde{z}_{2}, \tilde{v}\right)$.

Proof. The equilibria of model (2.1)-(2.4) satisfy the following equations:

$$
\lambda_{i}-d_{i} x_{i}-\frac{\beta_{i} x_{i} v}{\left(1+\mu_{i} x_{i}\right)\left(1+\alpha_{i} v\right)}=0
$$




$$
\begin{aligned}
\left(1-q_{i}\right) \frac{\beta_{i} x_{i} v}{\left(1+\mu_{i} x_{i}\right)\left(1+\alpha_{i} v\right)}-\delta_{i} y_{i} & =0, \\
q_{i} \frac{\beta_{i} x_{i} v}{\left(1+\mu_{i} x_{i}\right)\left(1+\alpha_{i} v\right)}-a_{i} z_{i} & =0, \\
\sum_{i=1}^{2}\left(N_{i} \delta_{i} y_{i}+M_{i} a_{i} z_{i}\right)-u v & =0 .
\end{aligned}
$$

From Eqs. (3.2) and (3.3) we have

$$
y_{i}=\frac{\left(1-q_{i}\right) \beta_{i} x_{i}}{\delta_{i}\left(1+\mu_{i} x_{i}\right)\left(1+\alpha_{i} v\right)} v, \quad z_{i}=\frac{q_{i} \beta_{i} x_{i}}{a_{i}\left(1+\mu_{i} x_{i}\right)\left(1+\alpha_{i} v\right)} v .
$$

Inserting $y_{i}$ and $z_{i}$ into Eq. (3.4) we obtain

$$
\left(\sum_{i=1}^{2} \frac{\left(\left(1-q_{i}\right) N_{i}+q_{i} M_{i}\right) \beta_{i} x_{i}}{u\left(1+\mu_{i} x_{i}\right)\left(1+\alpha_{i} v\right)}-1\right) u v=0 .
$$

Equation (3.6) has two possible solutions

$$
v=0 \text { or } \sum_{i=1}^{2} \frac{\left(\left(1-q_{i}\right) N_{i}+q_{i} M_{i}\right) \beta_{i} x_{i}}{u\left(1+\mu_{i} x_{i}\right)\left(1+\alpha_{i} v\right)}-1=0 .
$$

If $v=0$ then substituting it in Eqs. (3.1) and (3.5), leads to a disease-free equilibrium $E_{0}=\left(x_{1}^{0}, 0,0, x_{2}^{0}, 0,0,0\right)$, where $x_{i}^{0}=\frac{\lambda_{i}}{d_{i}}, i=1,2$.

If $v \neq 0$, then solving Eq. (3.1) w.r.t. $x_{i}$ we get:

$$
\begin{gathered}
x_{i}^{ \pm}= \\
\frac{\left[\left(\mu_{i} x_{i}^{0}-1\right)\left(1+\alpha_{i} v\right)-\frac{\beta_{i} v}{d_{i}}\right] \pm \sqrt{\left[\left(\mu_{i} x_{i}^{0}-1\right)\left(1+\alpha_{i} v\right)-\frac{\beta_{i} v}{d_{i}}\right]^{2}+4 \mu_{i} x_{i}^{0}\left(\left(1+\alpha_{i} v\right)^{2}\right.}}{2 \mu_{i}\left(1+\alpha_{i} v\right)},
\end{gathered}
$$

where $x_{i}^{0}=\frac{\lambda_{i}}{d_{i}}, i=1,2$. Clearly if $v>0$ then $x_{i}^{-}<0$ and $x_{i}^{+}>0$, then we choose $x_{i}=x_{i}^{+}$

$$
\begin{gathered}
x_{i}= \\
\frac{\left[\left(\mu_{i} x_{i}^{0}-1\right)\left(1+\alpha_{i} v\right)-\frac{\beta_{i} v}{d_{i}}\right]+\sqrt{\left[\left(\mu_{i} x_{i}^{0}-1\right)\left(1+\alpha_{i} v\right)-\frac{\beta_{i} v}{d_{i}}\right]^{2}+4 \mu_{i} x_{i}^{0}\left(1+\alpha_{i} v\right)^{2}}}{2 \mu_{i}\left(1+\alpha_{i} v\right)} .
\end{gathered}
$$

From Eqs. (3.1), (3.4) and (3.5) we get

$$
\sum_{i=1}^{2}\left(\left(1-q_{i}\right) N_{i}+q_{i} M_{i}\right)\left(\lambda_{i}-d_{i} x_{i}\right)-u v=0 .
$$


Since $x_{i}$ is a function of $v$, then we can define a function $H(v)$ as:

$$
H(v)=\sum_{i=1}^{2}\left(\left(1-q_{i}\right) N_{i}+q_{i} M_{i}\right)\left(\lambda_{i}-d_{i} x_{i}(v)\right)-u v=0 .
$$

Now, we want to show that there exist $\tilde{v}>0$ such that $H(\tilde{v})=0$. It is clear that, if $v=0$ then $x_{i}=x_{i}^{0}$ and $H(0)=0$, and when

$$
v=\bar{v}=\sum_{i=1}^{2} \frac{\left(\left(1-q_{i}\right) N_{i}+q_{i} M_{i}\right) \lambda_{i}}{u}>0,
$$

then we have $\bar{x}_{i}=x_{i}(\bar{v})>0$ and

$$
H(\bar{v})=-\sum_{i=1}^{2}\left(\left(1-q_{i}\right) N_{i}+q_{i} M_{i}\right) d_{i} \bar{x}_{i}<0 .
$$

Since $H(v)$ is continuous for all $v \geq 0$, we have that

$$
\begin{gathered}
H^{\prime}(0)= \\
\sum_{i=1}^{2} \frac{\left(\left(1-q_{i}\right) N_{i}+q_{i} M_{i}\right) \beta_{i} x_{i}^{0}}{\left(1+\mu_{i} x_{i}^{0}\right)}-u=u\left(\sum_{i=1}^{2} \frac{\left(\left(1-q_{i}\right) N_{i}+q_{i} M_{i}\right) \beta_{i} x_{i}^{0}}{u\left(1+\mu_{i} x_{i}^{0}\right)}-1\right) .
\end{gathered}
$$

Therefore, if $\sum_{i=1}^{2} \frac{\left(\left(1-q_{i}\right) N_{i}+q_{i} M_{i}\right) \beta_{i} x_{i}^{0}}{u\left(1+\mu_{i} x_{i}^{0}\right)}>1$, then $H^{\prime}(0)>0$, and there exists $\tilde{v} \in(0, \bar{v})$ such that $H(\tilde{v})=0$. From Eqs. (3.5) and (3), we have $\tilde{x}_{i}>0, \tilde{y}_{i}>0$ and $\tilde{z}_{i}>0$, $i=1,2$. Therefore, an endemic equilibrium $E_{1}=\left(\tilde{x}_{1}, \tilde{y}_{1}, \tilde{z}_{1}, \tilde{x}_{2}, \tilde{y}_{2}, \tilde{z}_{2}, \tilde{v}\right)$ exists when $\sum_{i=1}^{2} \frac{\left(\left(1-q_{i}\right) N_{i}+q_{i} M_{i}\right) \beta_{i} x_{i}^{0}}{u\left(1+\mu_{i} x_{i}^{0}\right)}>1$.

Now, we are ready to define the parameter $R_{0}$ as:

$$
R_{0}=\sum_{i=1}^{2} R_{0 i}=\sum_{i=1}^{2} \frac{\left(\left(1-q_{i}\right) N_{i}+q_{i} M_{i}\right) \beta_{i} x_{i}^{0}}{u\left(1+\mu_{i} x_{i}^{0}\right)},
$$

where, $R_{01}$ represents the basic infection reproduction number of the HIV dynamics with CD4 ${ }^{+}$T cells (in the absence of macrophages) and $R_{02}$ represents the basic infection reproduction number of the HIV dynamics with macrophages (in the absence of CD4 ${ }^{+} \mathrm{T}$ cells), respectively. The parameter $R_{0}$ determines whether the infection can be established.

Here, we establish the global stability of the two equilibria of system (2.1)-(2.4) 
employing the direct Lyapunov method and LaSalle's invariance principle. The following function will be used throughout the paper $F:(0, \infty) \rightarrow[0, \infty)$ as:

$$
F(s)=s-1-\ln s .
$$

Theorem 1. The disease-free equilibrium $E_{0}$ of system (2.1)-(2.4) is GAS when $R_{0} \leq 1$.

Proof. Define Lyapunov functional $W_{0}$ as:

$$
\sum_{i=1}^{2} \gamma_{i}\left[x_{i}=x_{i}^{0}-\int_{x_{i}^{0}}^{x_{i}} \frac{x_{i}^{0}\left(1+\mu_{i} s\right)}{s\left(1+\mu_{i} x_{i}^{0}\right)} d s+\frac{N_{i}}{\left(1-q_{i}\right) N_{i}+q_{i} M_{i}} y_{i}+\frac{M_{i}}{\left(1-q_{i}\right) N_{i}+q_{i} M_{i}} z_{i}\right]+v,
$$

where $\gamma_{i}=\left(1-q_{i}\right) N_{i}+q_{i} M_{i}, i=1,2$.

We calculate $\frac{d W_{0}}{d t}$ along the trajectories of system of (2.1)-(2.4) as:

$$
\begin{aligned}
\frac{d W_{0}}{d t} & =\sum_{i=1}^{2} \gamma_{i}\left[\left(1-\frac{x_{i}^{0}\left(1+\mu_{i} x_{i}\right)}{x_{i}\left(1+\mu_{i} x_{i}^{0}\right)}\right)\left(\lambda_{i}-d_{i} x_{i}-\frac{\beta_{i} x_{i} v}{\left(1+\mu_{i} x_{i}\right)\left(1+\alpha_{i} v\right)}\right)\right. \\
& +\frac{N_{i}}{\left(1-q_{i}\right) N_{i}+q_{i} M_{i}}\left(\frac{\left(1-q_{i}\right) \beta_{i} x_{i} v}{\left(1+\mu_{i} x_{i}\right)\left(1+\alpha_{i} v\right)}-\delta_{i} y_{i}\right) \\
& \left.+\frac{M_{i}}{\left(1-q_{i}\right) N_{i}+q_{i} M_{i}}\left(\frac{q_{i} \beta_{i} x_{i} v}{\left(1+\mu_{i} x_{i}\right)\left(1+\alpha_{i} v\right)}-a_{i} z_{i}\right)\right] \\
& +\sum_{i=1}^{2}\left(N_{i} \delta_{i} y_{i}+M_{i} a_{i} z_{i}\right)-u v
\end{aligned}
$$

Collecting terms of Eq. (3.11) we get

$$
\begin{aligned}
\frac{d W_{0}}{d t} & =\sum_{i=1}^{2} \gamma_{i}\left[d_{i}\left(1-\frac{x_{i}^{0}\left(1+\mu_{i} x_{i}\right)}{x_{i}\left(1+\mu_{i} x_{i}^{0}\right)}\right)\left(x_{i}^{0}-x_{i}\right)+\frac{\beta_{i} x_{i}^{0} v}{\left(1+\mu_{i} x_{i}^{0}\right)\left(1+\alpha_{i} v\right)}\right]-u v \\
& =-\sum_{i=1}^{2} \gamma_{i} \frac{d_{i}\left(x_{i}-x_{i}^{0}\right)^{2}}{x_{i}\left(1+\mu_{i} x_{i}^{0}\right)}-u v+u v \sum_{i=1}^{2} \frac{R_{0 i}}{\left(1+\alpha_{i} v\right)} \\
& =-\sum_{i=1}^{2} \gamma_{i} \frac{d_{i}\left(x_{i}-x_{i}^{0}\right)^{2}}{x_{i}\left(1+\mu_{i} x_{i}^{0}\right)}-\sum_{i=1}^{2} \frac{R_{0 i} \alpha_{i} u v^{2}}{\left(1+\alpha_{i} v\right)}+\left(R_{0}-1\right) u v .
\end{aligned}
$$

Then $\frac{d W_{0}}{d t} \leq 0$ for all $x_{1}, x_{2}, v>0$ when $R_{0} \leq 1$. We note that, the solutions of system (2.1)-(2.4) converge to $\Gamma$, the largest invariant subset of $\left\{\frac{d W_{0}}{d t}=0\right\}$. From Eq. (3.12), we have $\frac{d W_{0}}{d t}=0$ if and only if $x_{i}=x_{i}^{0}, i=1,2$ and $v=0$. The set $\Gamma$ 
is invariant and for any element belongs to $\Gamma$ satisfies $v=0$ and then $\dot{v}=0$. We can see from Eq. (2.4) that

$$
\sum_{i=1}^{2}\left(N_{i} \delta_{i} y_{i}+M_{i} a_{i} z_{i}\right)=0 .
$$

Since $y_{i}$ and $z_{i}$ are non-negative for $i=1,2$, then $y_{i}=z_{i}=0, i=1,2$. It follows that, $\frac{d W_{0}}{d t}=0$ iff $x_{i}=x_{i}^{0}, y_{i}=0, z_{i}=0, i=1,2$ and $v=0$. From LaSalle's invariance principle, $E_{0}$ is GAS.

Theorem 2. The endemic equilibrium $E_{1}$ of system (2.1)-(2.4) is GAS when $R_{0}>$ 1.

Proof. We consider the Lyapunov functional $W_{1}$ as:

$$
\begin{aligned}
W_{1} & =\sum_{i=1}^{2} \gamma_{i}\left[x_{i}-\tilde{x}_{i}-\int_{\tilde{x}_{i}}^{x_{i}} \frac{\tilde{x}_{i}\left(1+\mu_{i} s\right)}{s\left(1+\mu_{i} \tilde{x}_{i}\right)} d s+\frac{N_{i}}{\left(1-q_{i}\right) N_{i}+q_{i} M_{i}} \tilde{y}_{i} F\left(\frac{y_{i}}{\tilde{y}_{i}}\right)\right. \\
& \left.+\frac{M_{i}}{\left(1-q_{i}\right) N_{i}+q_{i} M_{i}} \tilde{z}_{i} F\left(\frac{z_{i}}{\tilde{z}_{i}}\right)\right]+\tilde{v} F\left(\frac{v}{\tilde{v}}\right) .
\end{aligned}
$$

Calculating $\frac{d W_{1}}{d t}$ along the solutions of system (2.1)-(2.4) we obtain

$$
\begin{aligned}
\frac{d W_{1}}{d t} & =\sum_{i=1}^{2} \gamma_{i}\left[\left(1-\frac{\tilde{x}_{i}\left(1+\mu_{i} x_{i}\right)}{x_{i}\left(1+\mu_{i} \tilde{x}_{i}\right)}\right)\left(\lambda_{i}-d_{i} x_{i}-\frac{\beta_{i} x_{i} v}{\left(1+\mu_{i} x_{i}\right)\left(1+\alpha_{i} v\right)}\right)\right. \\
& +\frac{N_{i}}{\left(1-q_{i}\right) N_{i}+q_{i} M_{i}}\left(1-\frac{\tilde{y}_{i}}{y_{i}}\right)\left(\frac{\left(1-q_{i}\right) \beta_{i} x_{i} v}{\left(1+\mu_{i} x_{i}\right)\left(1+\alpha_{i} v\right)}-\delta_{i} y_{i}\right) \\
& \left.+\frac{M_{i}}{\left(1-q_{i}\right) N_{i}+q_{i} M_{i}}\left(1-\frac{\tilde{z}_{i}}{z_{i}}\right)\left(\frac{q_{i} \beta_{i} x_{i} v}{\left(1+\mu_{i} x_{i}\right)\left(1+\alpha_{i} v\right)}-a_{i} z_{i}\right)\right] \\
& +\left(1-\frac{\tilde{v}}{v}\right)\left(\sum_{i=1}^{2}\left(N_{i} \delta_{i} y_{i}+M_{i} a_{i} z_{i}\right)-u v\right) .
\end{aligned}
$$

Collecting terms of Eq. (3.13) we get

$$
\begin{aligned}
\frac{d W_{1}}{d t} & =\sum_{i=1}^{2} \gamma_{i}\left[\left(1-\frac{\tilde{x}_{i}\left(1+\mu_{i} x_{i}\right)}{x_{i}\left(1+\mu_{i} \tilde{x}_{i}\right)}\right)\left(\lambda_{i}-d_{i} x_{i}\right)+\frac{\beta_{i} \tilde{x}_{i} v}{\left(1+\mu_{i} \tilde{x}_{i}\right)\left(1+\alpha_{i} v\right)}\right. \\
& -\frac{\left(1-q_{i}\right) N_{i}}{\left(1-q_{i}\right) N_{i}+q_{i} M_{i}} \frac{\tilde{y}_{i}}{y_{i}} \frac{\beta_{i} x_{i} v}{\left(1+\mu_{i} x_{i}\right)\left(1+\alpha_{i} v\right)}+\frac{N_{i} \delta_{i}}{\left(1-q_{i}\right) N_{i}+q_{i} M_{i}} \tilde{y}_{i} \\
& \left.-\frac{q_{i} M_{i}}{\left(1-q_{i}\right) N_{i}+q_{i} M_{i}} \frac{\tilde{z}_{i}}{z_{i}} \frac{\beta_{i} x_{i} v}{\left(1+\mu_{i} x_{i}\right)\left(1+\alpha_{i} v\right)}+\frac{M_{i} a_{i}}{\left(1-q_{i}\right) N_{i}+q_{i} M_{i}} \tilde{z}_{i}\right]
\end{aligned}
$$




$$
-u v-\frac{\tilde{v}}{v} \sum_{i=1}^{2} N_{i} \delta_{i} y_{i}-\frac{\tilde{v}}{v} \sum_{i=1}^{2} M_{i} a_{i} z_{i}+u \tilde{v} .
$$

Using the equilibrium conditions for $E_{1}$ :

$$
\begin{gathered}
\lambda_{i}=d_{i} \tilde{x}_{i}+\frac{\beta_{i} \tilde{x}_{i} \tilde{v}}{\left(1+\mu_{i} \tilde{x}_{i}\right)\left(1+\alpha_{i} \tilde{v}\right)}, \quad \frac{\left(1-q_{i}\right) \beta_{i} \tilde{x}_{i} \tilde{v}}{\left(1+\mu_{i} \tilde{x}_{i}\right)\left(1+\alpha_{i} \tilde{v}\right)}=\delta_{i} \tilde{y}_{i}, \\
\frac{q_{i} \beta_{i} \tilde{x}_{i} \tilde{v}}{\left(1+\mu_{i} \tilde{x}_{i}\right)\left(1+\alpha_{i} \tilde{v}\right)}=a_{i} \tilde{z}_{i}, \quad u \tilde{v}=\sum_{i=1}^{2}\left(N_{i} \delta_{i} \tilde{y}_{i}+M_{i} a_{i} \tilde{z}_{i}\right),
\end{gathered}
$$

and the following equality

$$
u v=u \tilde{v} \frac{v}{\tilde{v}}=\frac{v}{\tilde{v}}\left(\sum_{i=1}^{2}\left(N_{i} \delta_{i} \tilde{y}_{i}+M_{i} a_{i} \tilde{z}_{i}\right)\right)=\frac{v}{\tilde{v}} \sum_{i=1}^{2} \gamma_{i} \frac{\beta_{i} \tilde{x}_{i} \tilde{v}}{\left(1+\mu_{i} \tilde{x}_{i}\right)\left(1+\alpha_{i} \tilde{v}\right)},
$$

we obtain

$$
\begin{aligned}
\frac{d W_{1}}{d t} & =\sum_{i=1}^{2} \gamma_{i}\left[-\frac{d_{i}\left(x_{i}-\tilde{x}_{i}\right)^{2}}{x_{i}\left(1+\mu_{i} \tilde{x}_{i}\right)}+\frac{\beta_{i} \tilde{x}_{i} \tilde{v}}{\left(1+\mu_{i} \tilde{x}_{i}\right)\left(1+\alpha_{i} \tilde{v}\right)}\left(1-\frac{\tilde{x}_{i}\left(1+\mu_{i} x_{i}\right)}{x_{i}\left(1+\mu_{i} \tilde{x}_{i}\right)}\right)\right. \\
& +\frac{\beta_{i} \tilde{x}_{i} \tilde{v}}{\left(1+\mu_{i} \tilde{x}_{i}\right)\left(1+\alpha_{i} \tilde{v}\right)}\left(\frac{v\left(1+\alpha_{i} \tilde{v}\right)}{\tilde{v}\left(1+\alpha_{i} v\right)}-\frac{v}{\tilde{v}}\right) \\
& +\frac{2 N_{i} \delta_{i}}{\left(1-q_{i}\right) N_{i}+q_{i} M_{i}} \tilde{y}_{i}+\frac{2 M_{i} a_{i}}{\left(1-q_{i}\right) N_{i}+q_{i} M_{i}} \tilde{z}_{i} \\
& -\frac{N_{i} \delta_{i} \tilde{y}_{i}}{\left(1-q_{i}\right) N_{i}+q_{i} M_{i}} \frac{x_{i} v \tilde{y}_{i}\left(1+\mu_{i} \tilde{x}_{i}\right)\left(1+\alpha_{i} \tilde{v}\right)}{\tilde{x}_{i} \tilde{v} y_{i}\left(1+\mu_{i} x_{i}\right)\left(1+\alpha_{i} v\right)} \\
& -\frac{M_{i} a_{i} \tilde{z}_{i}}{\left(1-q_{i}\right) N_{i}+q_{i} M_{i}} \frac{x_{i} v \tilde{z}_{i}\left(1+\mu_{i} \tilde{x}_{i}\right)\left(1+\alpha_{i} \tilde{v}\right)}{\tilde{x}_{i} \tilde{v} z_{i}\left(1+\mu_{i} x_{i}\right)\left(1+\alpha_{i} v\right)} \\
& \left.-\frac{N_{i} \delta_{i} \tilde{y}_{i}}{\left(1-q_{i}\right) N_{i}+q_{i} M_{i}} \frac{y_{i} \tilde{v}}{\tilde{y}_{i} v}-\frac{M_{i} a_{i} \tilde{z}_{i}}{\left(1-q_{i}\right) N_{i}+q_{i} M_{i}} \frac{z_{i} \tilde{v}}{\tilde{z}_{i} v}\right] .
\end{aligned}
$$

Eq. (3.14) can be rewritten as:

$$
\begin{aligned}
\frac{d W_{1}}{d t} & =\sum_{i=1}^{2} \gamma_{i}\left[-\frac{d_{i}\left(x_{i}-\tilde{x}_{i}\right)^{2}}{x_{i}\left(1+\mu_{i} \tilde{x}_{i}\right)}+\frac{\beta_{i} \tilde{x}_{i} \tilde{v}}{\left(1+\mu_{i} \tilde{x}_{i}\right)\left(1+\alpha_{i} \tilde{v}\right)}\left(\frac{v\left(1+\alpha_{i} \tilde{v}\right)}{\tilde{v}\left(1+\alpha_{i} v\right)}-\frac{v}{\tilde{v}}\right)\right. \\
& +\frac{N_{i} \delta_{i}}{\left(1-q_{i}\right) N_{i}+q_{i} M_{i}} \tilde{y}_{i}\left(3-\frac{\tilde{x}_{i}\left(1+\mu_{i} x_{i}\right)}{x_{i}\left(1+\mu_{i} \tilde{x}_{i}\right)}-\frac{x_{i} \tilde{y}_{i} v\left(1+\mu_{i} \tilde{x}_{i}\right)\left(1+\alpha_{i} \tilde{v}\right)}{\tilde{x}_{i} y_{i} \tilde{v}\left(1+\mu_{i} x_{i}\right)\left(1+\alpha_{i} v\right)}-\frac{y_{i} \tilde{v}}{\tilde{y}_{i} v}\right) \\
& \left.+\frac{M_{i} a_{i}}{\left(1-q_{i}\right) N_{i}+q_{i} M_{i}} \tilde{z}_{i}\left(3-\frac{\tilde{x}_{i}\left(1+\mu_{i} x_{i}\right)}{x_{i}\left(1+\mu_{i} \tilde{x}_{i}\right)}-\frac{x_{i} \tilde{z}_{i} v\left(1+\mu_{i} \tilde{x}_{i}\right)\left(1+\alpha_{i} \tilde{v}\right)}{\tilde{x}_{i} z_{i} \tilde{v}\left(1+\mu_{i} x_{i}\right)\left(1+\alpha_{i} v\right)}-\frac{z_{i} \tilde{v}}{\tilde{z}_{i} v}\right)\right] \\
& =\sum_{i=1}^{2} \gamma_{i}\left[-\frac{d_{i}\left(x_{i}-\tilde{x}_{i}\right)^{2}}{x_{i}\left(1+\mu_{i} \tilde{x}_{i}\right)}\right.
\end{aligned}
$$




$$
\begin{aligned}
& +\frac{\beta_{i} \tilde{x}_{i} \tilde{v}}{\left(1+\mu_{i} \tilde{x}_{i}\right)\left(1+\alpha_{i} \tilde{v}\right)}\left(\frac{v\left(1+\alpha_{i} \tilde{v}\right)}{\tilde{v}\left(1+\alpha_{i} v\right)}-\frac{v}{\tilde{v}}-1+\frac{1+\alpha_{i} v}{1+\alpha_{i} \tilde{v}}\right) \\
& +\frac{N_{i} \delta_{i}}{\left(1-q_{i}\right) N_{i}+q_{i} M_{i}} \tilde{y}_{i}\left(4-\frac{\tilde{x}_{i}\left(1+\mu_{i} x_{i}\right)}{x_{i}\left(1+\mu_{i} \tilde{x}_{i}\right)}-\frac{x_{i} \tilde{y}_{i} v\left(1+\mu_{i} \tilde{x}_{i}\right)\left(1+\alpha_{i} \tilde{v}\right)}{\tilde{x}_{i} y_{i} \tilde{v}\left(1+\mu_{i} x_{i}\right)\left(1+\alpha_{i} v\right)}\right. \\
& \left.-\frac{y_{i} \tilde{v}}{\tilde{y}_{i} v}-\frac{1+\alpha_{i} v}{1+\alpha_{i} \tilde{v}}\right)+\frac{M_{i} a_{i}}{\left(1-q_{i}\right) N_{i}+q_{i} M_{i}} \tilde{z}_{i}\left(4-\frac{\tilde{x}_{i}\left(1+\mu_{i} x_{i}\right)}{x_{i}\left(1+\mu_{i} \tilde{x}_{i}\right)}\right. \\
& \left.\left.-\frac{x_{i} \tilde{z}_{i} v\left(1+\mu_{i} \tilde{x}_{i}\right)\left(1+\alpha_{i} \tilde{v}\right)}{\tilde{x}_{i} z_{i} \tilde{v}\left(1+\mu_{i} x_{i}\right)\left(1+\alpha_{i} v\right)}-\frac{z_{i} \tilde{v}}{\tilde{z}_{i} v}-\frac{1+\alpha_{i} v}{1+\alpha_{i} \tilde{v}}\right)\right] \\
& =\sum_{i=1}^{2} \gamma_{i}\left[-\frac{d_{i}\left(x_{i}-\tilde{x}_{i}\right)^{2}}{x_{i}\left(1+\mu_{i} \tilde{x}_{i}\right)}-\frac{\beta_{i} \tilde{x}_{i} \alpha_{i}(v-\tilde{v})^{2}}{\left(1+\mu_{i} \tilde{x}_{i}\right)\left(1+\alpha_{i} \tilde{v}\right)^{2}\left(1+\alpha_{i} v\right)}\right. \\
& +\frac{N_{i} \delta_{i}}{\left(1-q_{i}\right) N_{i}+q_{i} M_{i}} \tilde{y}_{i}\left(4-\frac{\tilde{x}_{i}\left(1+\mu_{i} x_{i}\right)}{x_{i}\left(1+\mu_{i} \tilde{x}_{i}\right)}-\frac{x_{i} \tilde{y}_{i} v\left(1+\mu_{i} \tilde{x}_{i}\right)\left(1+\alpha_{i} \tilde{v}\right)}{\tilde{x}_{i} y_{i} \tilde{v}\left(1+\mu_{i} x_{i}\right)\left(1+\alpha_{i} v\right)}\right. \\
& \left.-\frac{y_{i} \tilde{v}}{\tilde{y}_{i} v}-\frac{1+\alpha_{i} v}{1+\alpha_{i} \tilde{v}}\right)+\frac{M_{i} a_{i}}{\left(1-q_{i}\right) N_{i}+q_{i} M_{i}} \tilde{z}_{i}\left(4-\frac{\tilde{x}_{i}\left(1+\mu_{i} x_{i}\right)}{x_{i}\left(1+\mu_{i} \tilde{x}_{i}\right)}\right. \\
& \left.\left.-\frac{x_{i} \tilde{z}_{i} v\left(1+\mu_{i} \tilde{x}_{i}\right)\left(1+\alpha_{i} \tilde{v}\right)}{\tilde{x}_{i} z_{i} \tilde{v}\left(1+\mu_{i} x_{i}\right)\left(1+\alpha_{i} v\right)}-\frac{z_{i} \tilde{v}}{\tilde{z}_{i} v}-\frac{1+\alpha_{i} v}{1+\alpha_{i} \tilde{v}}\right)\right] \text {. }
\end{aligned}
$$

Because the geometrical mean is less than or equal to the arithmetical mean, then the last two terms of Eq. (3.15) are less than or equal zero. Therefore, if $R_{0}>1$, then $\tilde{x}_{1}, \tilde{x}_{2}, \tilde{y}_{1}, \tilde{y}_{2}, \tilde{z}_{1}, \tilde{z}_{2}, \tilde{v}>0$, and $\frac{d W_{1}}{d t} \leq 0$ for all $x_{1}, x_{2}, y_{1}, y_{2}, z_{1}, z_{2}, v>0$. It is clear that, the set $\left\{\frac{d W_{1}}{d t}=0\right\}$ contains only the invariant singleton set $\left\{E_{1}\right\}$. The global stability of $E_{1}$ is induced from LaSalle's invariance principle.

\section{NUMERICAL RESULTS AND DISCUSSIONS}

In this section, we use numerical simulations to illustrate theoretical results given in Theorems 1 and 2 for model (2.1)-(2.4). We shall fix the following parameters: $\lambda_{1}=10, \lambda_{2}=6, d_{1}=0.01, d_{2}=0.01, \delta_{1}=0.5, \delta_{2}=0.3, a_{1}=0.3, a_{2}=0.1, \alpha_{1}=$ $0.05, \alpha_{2}=0.05, \mu_{1}=0.0005, \mu_{2}=0.0005, N_{1}=20, N_{2}=10, M_{1}=10, M_{2}=5$, $\bar{\beta}_{1}=0.0005, \bar{\beta}_{2}=0.0008, q_{1}=q_{2}=0.5, f=0.5$ and $u=2$. The parameter $\varepsilon$ will be chosen below:

(i) $R_{0} \leq 1$. Let $\varepsilon=0.98$, then we get $R_{0}=0.88$. According to Theorem $1, E_{0}$ is GAS. Figures 1-7 show that the numerical results are compatible with the results of Theorem 1 . We can see that, the concentrations of uninfected $\mathrm{CD}^{+} \mathrm{T}$ cells and macrophages are increasing and converge to their normal values $\frac{\lambda_{1}}{d_{1}}=1000, \frac{\lambda_{2}}{d_{2}}=600$, respectively, while the concentrations of infected cells and free viruses are decaying and tend to zero. In this case, the treatment succeeded to eliminate the viruses from the blood. 
(ii) $R_{0}>1$. We choose $\varepsilon=0$, then we compute $R_{0}=4.73$. From Figures 1 7 we can see that, our simulation results are consistent with the theoretical results of Theorem 2, where is $E_{1}$ GAS. We can observe that, the trajectory of system (2.1)-(2.4) converges to $E_{1}=(630.16,3.69,6.16,300.38,4.99,14.98,38.97)$. We note that, when $\varepsilon=0$, i.e. there is no treatment the infection becomes chronic.

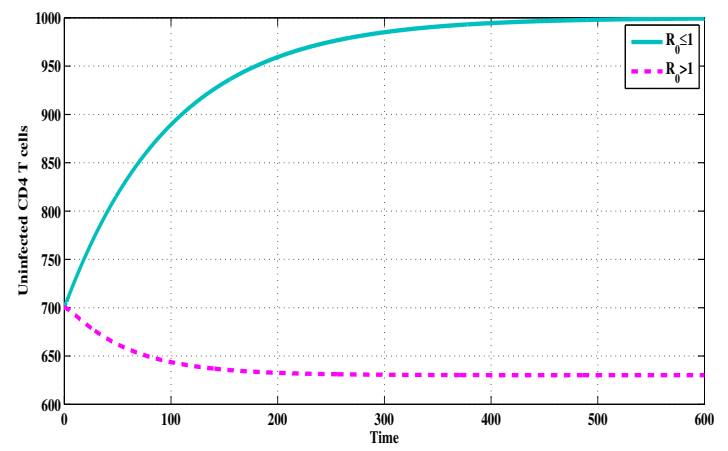

FIGURE 1. The evolution of uninfected $\mathrm{CD} 4^{+} \mathrm{T}$ cells for model (2.1)-(2.4).

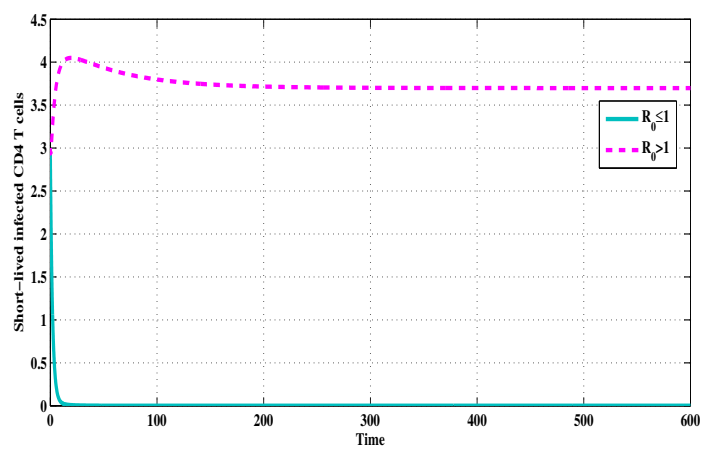

FIGURE 2. The evolution of short-lived infected $\mathrm{CD} 4^{+} \mathrm{T}$ cells for model (2.1)-(2.4).

\section{CONCLUSION}

In this paper, we have investigated the qualitative behavior of an HIV infection model which describes the interaction of the HIV with two classes of target cells, $\mathrm{CD} 4^{+} \mathrm{T}$ cells and macrophages with different drug efficacy. Both short-lived and 


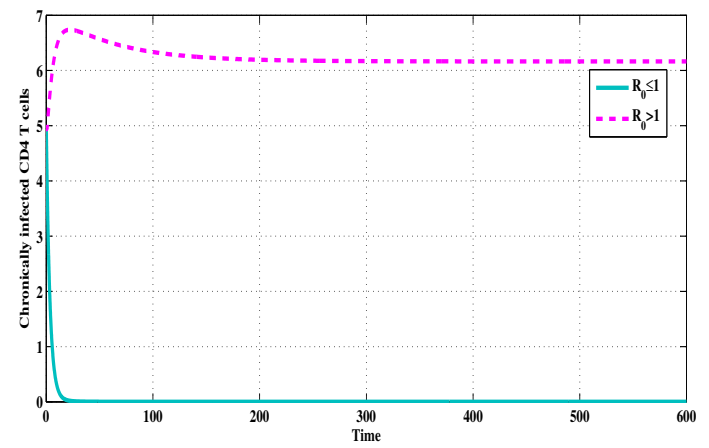

FIGURE 3. The evolution of chronically infected $\mathrm{CD} 4^{+} \mathrm{T}$ cells for model (2.1)-(2.4).

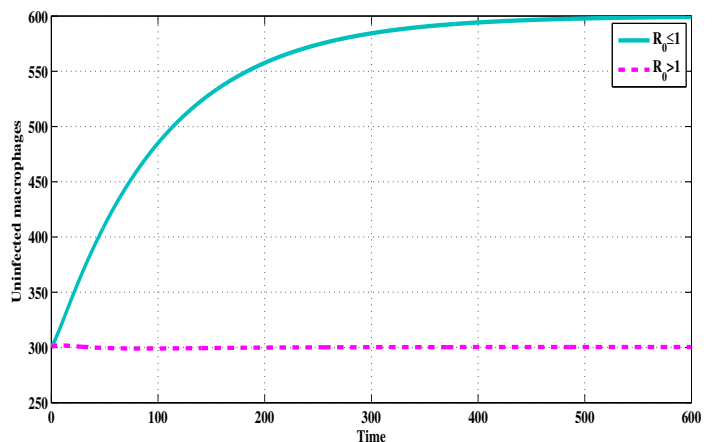

FIGURE 4. The evolution of uninfected macrophages cells for model (2.1)-(2.4).

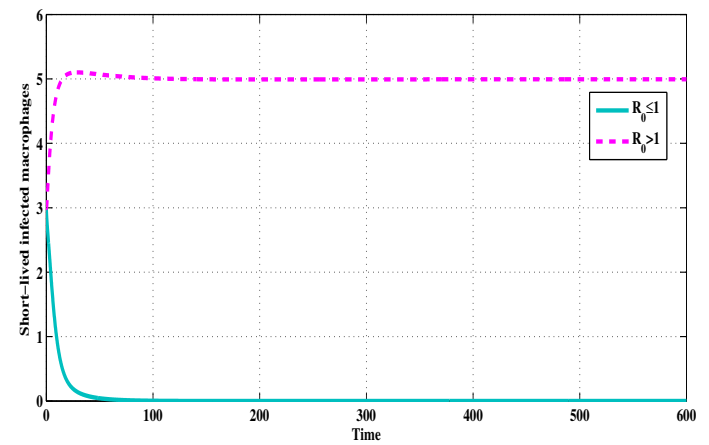

FIGURE 5. The evolution of short-lived infected macrophages cells for model (2.1)-(2.4). 


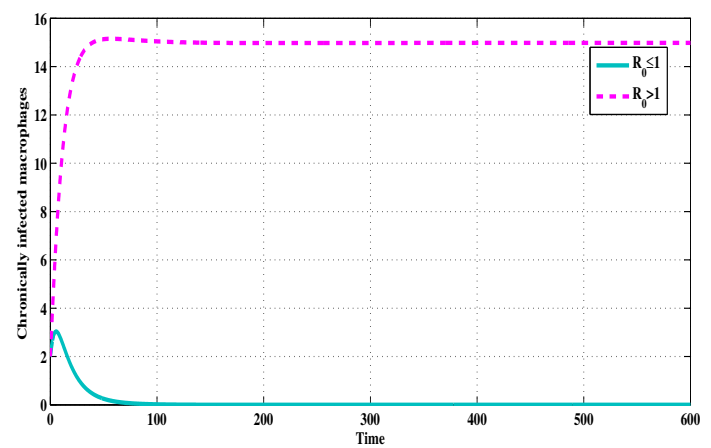

FIGURE 6. The evolution of chronically infected macrophages cells for model (2.1)-(2.4).

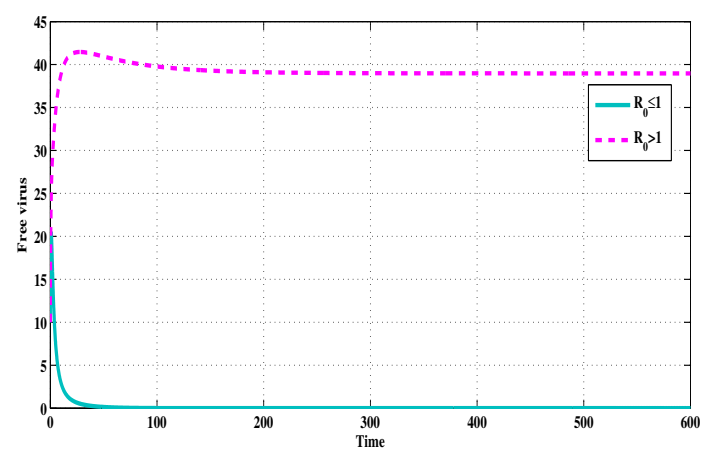

FIGURE 7. The evolution of free virus for model (2.1)-(2.4).

long lived chronically infected cells have been taken into account. The infection rate is given by Crowley-Martin functional response. The global stability of the diseasefree equilibrium and endemic equilibrium of the model has been established by constructing suitable Lyapunov functionals and using LaSalle's invariant principle. We have derived the basic infection reproduction number $R_{0}$ for the model. We have proven that, the disease-free equilibrium $E_{0}$ is GAS when $R_{0} \leq 1$, and the endemic equilibrium $E_{1}$ is GAS when $R_{0}>1$.

\section{ACKNOWLEDGEMENTS}

The authors are grateful to the anonymous reviewers for constructive suggestions and valuable comments, which improve the quality of the article. 


\section{REFERENCES}

[1] S. Bonhoeffer, R. M. May, G. Shaw, and M. A. Nowak, "Virus dynamics and drug therapy," Proceedings of the National Academy of Sciences of the United States of America, vol. 94, pp. 6971-6976, 1997.

[2] D. S. Callaway and A. S. Perelson, "HIV-1 infection and low steady state viral loads," Bulletin of Mathematical Biology, vol. 64, pp. 29-64, 2002, doi: 10.1006/bulm.2001.0266.

[3] A. M. Elaiw, "Global dynamics of an HIV infection model with two classes of target cells and distributed delays," Discrete Dynamics in Nature and Society, pp. 2425-2432, 2012.

[4] A. M. Elaiw, "Global properties of a class of virus infection models with multitarget cells," Nonlinear Dynamics, vol. 69, pp. 423-435, 2012, doi: 10.1007/s11071-011-0275-0.

[5] A. M. Elaiw, I. Hassanien, and S. A. Azoz, "Global stability of HIV infection models with intracellular delays," Journal of the Korean Mathematical Society, vol. 49, no. 4, pp. 779-794, 2012, doi: 10.4134/JKMS.2012.49.4.779.

[6] A. M. Elaiw and X. Xia, "HIV dynamics: Analysis and robust multirate MPC-based treatment schedules," Journal of Mathematical Analysis and Applications, vol. 356, pp. 285-301, 2009, doi: 10.1016/j.jmaa.2009.05.038.

[7] G. Huang, W. Ma, and Y. Takeuchi, "Global properties for virus dynamics model with BeddingtonDeAngelis functional response,” Applied Mathematics Letters, vol. 22, pp. 1690-1693, 2009, doi: 10.1016/j.aml.2009.06.004.

[8] M. A. Obaid and A. M. Elaiw, "Stability of virus infection models with antibodies and chronically infected cells," Abstract and Applied Analysis, vol. 217, pp. 2425-2432, 2014, Article ID 650371, doi: $10.1155 / 2014 / 650371$.

[9] A. S. Perelson, P. Essunger, and Y. C. et al., "Decay characteristics of HIV-1-infected compartments during combination therapy," Nature, vol. 397, no. 6629, pp. 188-191, 1997, doi: 10.1038/387188a0.

[10] A. S. Perelson, D. Kirschner, and R. D. Boer, "Dynamics of HIV infection of CD4 ${ }^{+}$T cells," Mathematical Biosciences, vol. 114, no. 1, pp. 81-125, 1993, doi: 10.1016/0025-5564(93)90043A.

[11] X. Wang, Y. Tao, and X. Song, "Delayed HIV-1 infection model Beddington-DeAngelis functional response," Nonlinear Dynamics, vol. 62, no. 1-2, pp. 67-72, 2009, doi: 10.1007/s11071-0109699-1.

[12] S. Xu, "Global stability of the virus dynamics model with Crowley-Martin functional response," Electronic Journal of Qualitative Theory of Differential Equations, vol. 9, pp. 1-10, 2012.

[13] X. Zhou and J. Cui, "Global stability of the viral dynamics with Crowley-Martin functional response," Bulletin of Kerala Mathematics Association, vol. 48, pp. 555-574, 2011.

Authors' addresses

A. M. Elaiw

King Abdulaziz University, Department of Mathematics, Faculty of Science, P.O. Box 80203, Jeddah 21589, Saudi Arabia

E-mail address: a_m_elaiw@yahoo.com

N. A. Almuallem

King Abdulaziz University, Department of Mathematics, Faculty of Science, P.O. Box 80203, Jeddah 21589, Saudi Arabia

Current address: King Abdulaziz University, Department of Mathematics, Faculty of Science, P. O. Box 50505, Jeddah, Saudi Arabia

E-mail address: nalmuallemegmail.com 